\title{
Dimensionality-tuned electronic structure of nickelate superlattices explored by soft-x-ray angle-resolved photoelectron spectroscopy
}

\author{
G. Berner, ${ }^{1}$ M. Sing, ${ }^{1}$ F. Pfaff, ${ }^{1}$ E. Benckiser, ${ }^{2}$ M. Wu, ${ }^{2}$ G. Christiani, ${ }^{2}$ G. Logvenov,${ }^{2}$ H.-U. Habermeier, ${ }^{2}$ M. Kobayashi, ${ }^{3}$ \\ V. N. Strocov, ${ }^{3}$ T. Schmitt, ${ }^{3}$ H. Fujiwara, ${ }^{4}$ S. Suga, ${ }^{5}$ A. Sekiyama, ${ }^{5}$ B. Keimer, ${ }^{2}$ and R. Claessen ${ }^{1}$ \\ ${ }^{1}$ Physikalisches Institut and Röntgen Center for Complex Material Systems (RCCM), Universität Würzburg, \\ Am Hubland, D-97074 Würzburg, Germany \\ ${ }^{2}$ Max Planck Institute for Solid State Research, Heisenbergstrasse 1, D-70569 Stuttgart, Germany \\ ${ }^{3}$ Swiss Light Source, Paul Scherrer Institut, CH-5232 Villigen, Switzerland \\ ${ }^{4}$ Division of Materials Physics, Graduate School of Engineering Science, Osaka University, Osaka 560-8531, Japan \\ ${ }^{5}$ Institute of Scientific \& Industrial Research, Osaka University, Ibaraki, Osaka 567-0047, Japan \\ (Received 21 November 2014; revised manuscript received 6 February 2015; published 16 September 2015)
}

\begin{abstract}
The electronic and magnetic properties of epitaxial $\mathrm{LaNiO}_{3} / \mathrm{LaAlO}_{3}$ superlattices can be tuned by layer thickness and substrate-induced strain. Here, we report on direct measurements of the $k$-space-resolved electronic structure of buried nickelate layers in superlattices under compressive strain by soft-x-ray photoemission. After disentangling strong extrinsic contributions to the angle-dependent signal caused by photoelectron diffraction, we are able to extract Fermi surface information from our data. We find that with decreasing $\mathrm{LaNiO}_{3}$ thickness down to two unit cells ( 2 uc) quasiparticle coherence becomes strongly reduced, in accord with the dimensioninduced metal-to-insulator transition seen in transport measurements. Nonetheless, on top of a strongly incoherent background a residual Fermi surface can be identified in the 2 uc superlattice whose nesting properties are consistent with the spin-density-wave (SDW) instability recently reported. The overall behavior of the Ni $3 d$ spectra and the absence of a complete gap opening indicate that the SDW phase is dominated by strong order parameter fluctuations.
\end{abstract}

DOI: 10.1103/PhysRevB.92.125130

PACS number(s): 79.60.Jv, 73.20.-r, 73.50.Pz

\section{INTRODUCTION}

Artificial transition metal oxide heterostructures potentially host novel electronic and magnetic phases not present in the bulk form of the constituents. Such phases may be controlled by, e.g., elastic strain or interfacial charge transfer [1]. In this context the perovskite $\mathrm{LaNiO}_{3}$ (LNO) is a very interesting material, as it is the only member of the $R E \mathrm{NiO}_{3}(R E=$ rare earth ion) family showing metallic behavior with its partially filled degenerate $\mathrm{Ni} 3 d e_{g}\left(d_{z^{2}}\right.$ and $\left.d_{x^{2}-y^{2}}\right)$ orbitals, while the other compounds exhibit a correlation-driven metalinsulator (MI) transition at low temperatures [2,3]. LNO is thus a highly correlated metal being close to an insulating phase, and therefore is susceptible to changes induced by heterostructuring.

Indeed, recent investigations have shown that the physical properties of LNO can be tuned by reducing its dimensionality or applying strain. For instance, density functional theory (DFT) calculations found that a single unit cell (uc) of LNO sandwiched between layers of the band insulator $\mathrm{LaAlO}_{3}$ (LAO) and thus under tensile strain displays a cuprate-like Fermi surface, identifying LNO as a possible candidate for exotic superconductivity [4-6]. Inspired by these predictions a large number of experimental and theoretical studies have been performed on ultrathin films and superlattices (SLs) [7-21].

Experimentally, ultrathin films are found to exhibit a dimensional crossover from metallic to insulating behavior upon reducing the film thickness, with critical values ranging from 3 to 9 uc [7-10]. The experimental observations for LNObased SLs are similar: while thicker LNO layers embedded in SL structures with a large band-gap insulator remain metallic at all temperatures, 2 uc thick layers show a temperaturedependent MI transition [12], accompanied by the onset of noncollinear antiferromagnetic order [14]. The epitaxial strain induced by the substrate only slightly affects the transition temperature, but a lifting of the $e_{g}$ orbital degeneracy is observed which depends on the sign of strain. While for compressive strain a lowering of the $d_{z^{2}}$ orbital is observed, in case of tensile strain the $d_{x^{2}-y^{2}}$ orbital is lower in energy. Note that for LNO thin films an asymmetric orbital-lattice coupling has been reported [13]. Besides the orbital energies, also the occupancies of the $\mathrm{Ni} 3 d$ orbitals, i.e., the orbital polarization, can be changed in superlattices built from two material components. It increases almost linearly with the induced strain up to a value of $25 \%$ [17,18]. Very recently, it has been suggested that new approaches and superlattice designs based on three material components are needed to enhance the orbital polarization beyond this value [22]. The reason for the appearance of the insulating state in both SLs and ultrathin films is still under debate and possibly not related. Different scenarios such as Anderson localization [7,8], Mott insulator transition [5,6], charge disproportionation [13,23], and spin density wave $[12,17]$ have been discussed.

We would like to emphasize that in superlattices the necessity of octahedral connectivity across the interfaces in combination with strain induced by the substrate can stabilize different distortions compared to the situation in epitaxial films [24,25]. Recent x-ray diffraction and electron microscopy studies demonstrated that the structural properties of LNO-based superlattices are clearly distinct from their thin-film counterparts [24-29]. The structural differences essentially appear in different $\mathrm{NiO}_{6}$ octahedral rotation patterns and/or angles resulting in different $\mathrm{Ni}-\mathrm{O}$ bond distances and different Ni-O-Ni bond angles. Due to the strong structure-property relationship within the $R E \mathrm{NiO}_{3}$ family, 
the electronic and magnetic properties are very sensitive to such small structural changes. Theoretical studies even argued that for $R E=\operatorname{Pr}$ and Nd nickelates the orthorhombic crystal symmetry is essential for the stabilization of charge order and that the charge disproportionation is proportional to the magnitude of the orthorhombic distortion [20,21]. Furthermore, the impact of the surface of thin films is not negligible in the ultrathin limit, in particular, when studied by surface-sensitive experiments. Since LNO with its $\mathrm{LaO}^{+}$ and $\mathrm{NiO}_{2}^{-}$sublayers is polar, the (001)-oriented free surfaces of thin LNO films tend to reconstruct structurally by polar distortions or octahedral rotations to minimize the electrostatic energy $[25,26,30]$. The modified structure results in a change of the electronic structure, at least near the surface. In contrast, the LNO layers in the SLs are embedded in LAO, a host material of like polarity, and are thus stabilized against structural effects resulting from polar discontinuities, as shown by x-ray diffraction [30]. Thus, the physical properties of the strained LNO layers and their dependence on dimensionality can be studied under clean conditions, i.e., in the absence of additional ionic or electronic surface and interface reconstructions. These differences to thin films make it necessary to experimentally study the electronic structure of buried layers in a superlattice and to track changes of the Fermi surface topology related to the confinement and the different structural distortions.

Direct insight into the electronic structure can be achieved by photoelectron spectroscopy (PES). Many of the scenarios mentioned above are reflected directly or indirectly in the microscopic electronic structure, i.e., in the singleparticle spectral function, to which PES provides direct access. Such investigations have focused so far on (ultrathin) films by angle-integrated [9] as well as angle-resolved PES (ARPES) [10,31,32]. However, ARPES studies on SLs require a more bulk-sensitive approach, since the LNO layers are buried below several unit cells of the host material (LAO in our case). Conventional photoemission with low-energy photons is limited by its very low information depth of a few angstroms only, determined by the photoelectron mean-free path (MFP), and therefore cannot easily access the LNO layers. In contrast, photons in the soft $\mathrm{X}$-ray (SX) regime allow for higher MFP at still reasonable momentum resolution ( $k$ resolution), thereby enabling $k$-resolved probing of the buried electronic structure in such SLs $[33,34]$.

In this study, we present SX-ARPES measurements on LNO/LAO SLs under compressive strain and a detailed analysis of the buried electronic structure to shed light on the nature of the temperature-dependent MI transition observed in SLs with 2 uc thick LNO layers and in particular the insulating phase. Besides a significant loss of quasiparticle (QP) coherence near the Fermi level $\left(E_{F}\right)$ for the two-dimensional (2D) ground state, observed in the angle-integrated valence band spectra, our $k$-resolved measurements trace the dimensional crossover from a three-dimensional (3D) Fermi surface in the $4 \mathrm{uc}$ LNO-SL to 2D behavior in the $2 \mathrm{uc} \mathrm{LNO}-\mathrm{SL}$. Although the intensity distribution in our $k$-space maps is strongly affected by x-ray photoelectron diffraction (XPD), we are able to extract band structure information on the $\mathrm{Ni} d_{x^{2}-y^{2}}$-derived hole pocket states in all LNO/LAO SLs. An indication of the $\mathrm{Ni} d_{z^{2}}$-derived electron pocket is only observed in the $2 \mathrm{uc}$ LNO SL. The residual Fermi surface observed in this sample displays strong nesting properties which are consistent with the spin-density-wave (SDW) scenario recently put forward as explanation for the insulating low-temperature ground state.

\section{EXPERIMENT}

LNO/LAO superlattices with different LNO layer thickness were grown by pulsed laser deposition on (001) $\mathrm{LaSrAlO}_{4}$ (LSAO) single-crystal substrates. The deposition starts with a number of $N(=2,4)$ unit cells of LNO followed by the same number of LAO layers. This stacking sequence $(N / / N)$ is repeated fifteen and eight times for $N=2,4$, respectively, terminating with an LAO layer at the surface [see Fig. 1(a)]. Additionally, a $(10 / / 4) \times 4$ SL was prepared being used as a thick LNO reference layer, also capped by 4 uc LAO.

The LNO/LAO stacks are compressively strained to the LSAO substrate, since the lattice mismatch between the LSAO and bulk pseudocubic LNO ( $a=3.838 \AA$ ) [35] is $\approx-3.2 \%$ [18]. The induced biaxial strain, which is stable up to a SL thickness of $\approx 50 \mathrm{~nm}$ [36], was confirmed by (a)

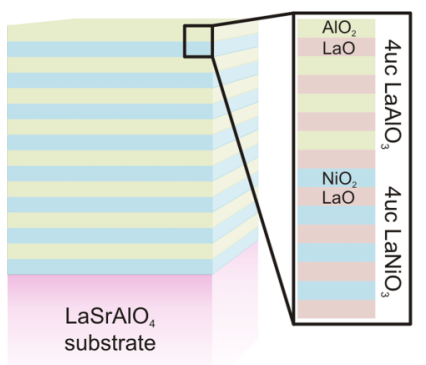

(b)

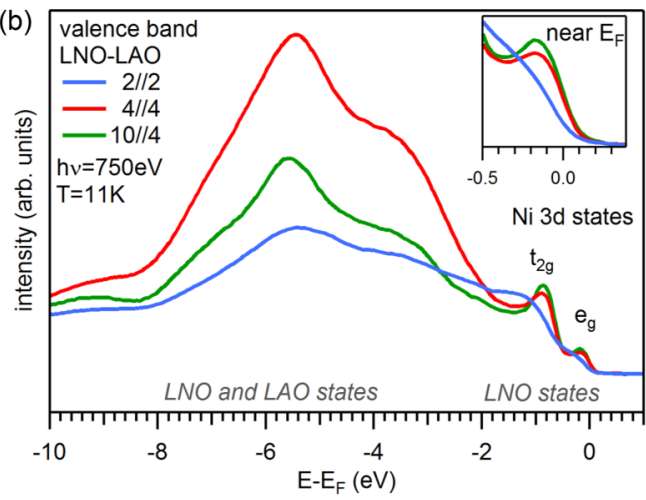

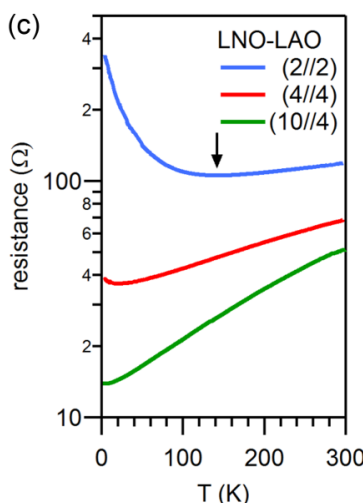

FIG. 1. (Color online) (a) Layout of the (4//4) LNO/LAO superlattice. The first LNO layer is buried below four uc LAO. (b) $k$-integrated valence band spectra of the investigated LNO/LAO samples at low temperature. The $(2 / / 2)$ SL exhibits a dramatic loss of quasiparticle coherence at the Fermi level (see inset). The spectra are normalized to the same integrated area between $-2 \mathrm{eV}$ and $E_{F}$ (without background subtraction). (c) Temperature-dependent resistivity measurements. The (2//2) SL shows a continuous MI transition at $T \approx 150 \mathrm{~K}$, while the $(4 / / 4)$ and $(10 / / 4)$ SLs stay metallic down to very low temperatures. 
X-ray diffraction with lattice parameters $a=3.750 \AA$ and $c=3.840 \AA$ [18]. Since, as pointed out in the introduction, the details of the structural distortions, in particular the $\mathrm{NiO}_{6}$ octahedral tilts and rotations are subtle and involved [28,36], we chose to perform the present photoemission experiments on superlattice structures identical to those used in previous studies $[11,12,17,18]$, reporting on results from various methods such as x-ray diffraction, resonant $\mathrm{x}$-ray scattering, ellipsometry, and Raman spectroscopy.

The photoemission experiments were performed at the ADRESS beamline of the Swiss Light Source with a SPECS Phoibos 150 spectrometer using $p$-polarized photons [34,37]. The overall energy resolution was $70 \mathrm{meV}$ at a photon energy of $700 \mathrm{eV}$. The angular resolution amounted to $0.07^{\circ}$ and $0.14^{\circ}$ along and perpendicular to the analyzer slit, respectively. During the experiment the samples were cooled down to $T=$ $11 \mathrm{~K}$. Preliminary SX-ARPES experiments were performed at beamline BL25SU, SPring-8, Japan, to establish the feasibility of the method. Prior to the measurements the sample surface was cleaned by keeping the samples under ozone flow for $45 \mathrm{~min}$, followed by an in situ annealing at $180{ }^{\circ} \mathrm{C}$ under $1 \times 10^{-5}$ mbar of oxygen for $45 \mathrm{~min}$. This method was found to strongly suppress the amount of carbon-containing surface contaminants.

\section{RESULTS}

\section{A. Angle-integrated spectra}

Figure 1(b) shows the angle-integrated valence band (VB) spectra of the $(2 / / 2),(4 / / 4)$, and $(10 / / 4)$ SLs measured in normal emission geometry at low temperature $(T=11 \mathrm{~K})$. Below $-2 \mathrm{eV}$ the valence band mainly consists of superposed LAO and LNO O $2 p$-derived states. Due to the valence band offset of $\approx 2 \mathrm{eV}$ between LAO and LNO (see Appendix A) the spectral weight between $-2 \mathrm{eV}$ and $E_{F}$ can unambiguously be assigned to LNO states, in particular to the crystal-field split $\mathrm{Ni}$ $3 d t_{2 g}$ and $e_{g}$ states at $\approx-0.8 \mathrm{eV}$ and at $E_{F}$, respectively. The octahedral splitting is consistent with values observed in thin LNO films $[9,15,38]$. The spectra are normalized to the same integrated area between $-2 \mathrm{eV}$ and $E_{F}$ (without background subtraction) in order to facilitate easy identification of the changes in the $\mathrm{Ni} 3 d$ states in this energy window. A normalization to the integral intensity of the full valence band (between $-10 \mathrm{eV}$ and $E_{F}$ ) would not provide a meaningful comparison because of the superimposed LAO and LNO valence band contributions, whose relative intensities strongly change for different layer thicknesses due to the finite probing depth.

In the VB spectra of the $(4 / / 4)$ and $(10 / / 4)$ SL the $e_{g}$-derived feature shows a clear cutoff by the Fermi-Dirac function at $E_{F}$ signaling that the samples remain metallic at low temperature down to a LNO layer thickness of 4 uc [see Fig. 1(b), inset]. This is consistent with electrical transport measurements shown in Fig. 1(c) and recent results from optical ellipsometry on the same SLs [12].

In comparison, the VB spectrum of the $(2 / / 2)$ SL displays a remarkable change near the Fermi level: No Fermi edge can be identified, instead both the $t_{2 g}$ and $e_{g}$ features are smeared out indicating a loss of quasiparticle coherence [see
Fig. 1(b), inset]. In contrast to a recent photoemission study of ultrathin films [9] our VB spectrum does not show a full band gap opening, but a distinct reduction of spectral weight at the Fermi level. Corresponding four-point-probe transport measurements of the identical samples confirm an insulating phase at low temperature and a temperature-dependent MI crossover at $T \approx 150 \mathrm{~K}$ driven by the reduced dimensionality [see arrow in Fig. 1(c)]. For very thin LNO layers such a temperature-dependent transition has only been observed in SL structures, but not in films [12,30].

\section{B. $k$-resolved spectra}

The angle-integrated spectra already demonstrate the pronounced effect of reduced LNO layer thickness on the electronic structure. In the following we study this in more detail by using the $k$-space resolved spectra, in particular with respect to Fermi surface (FS) volume and topology. For the interpretation of the measured data it is helpful to start the discussion from the theoretical FS obtained by DFT calculations. Figure 2 shows cuts through the expected 3D FS of bulk LNO parallel to the surface $\left(k_{z}=0 \AA^{-1}\right.$ and $\frac{\pi}{c}$, $k_{z}$ denoting the wave vector component perpendicular to the surface) as well as the $k_{z}$-independent 2D FS of a single LNO layer [calculated without (c) and with (d) correlation effects], based on Refs. [39] and [5], respectively. In the case of bulk LNO the Ni $d_{z^{2}}$ states form an electron pocket at the Brillouin zone (BZ) center ( $\Gamma$ point) [cf. Fig. 2(a)], while the Ni $d_{x^{2}-y^{2}}$ orbitals create large hole pockets at the zone corners (A points) [cf. Fig. 2(b)]. By reducing the LNO layer thickness, the 3D FS transforms into a 2D one, where both the electron and hole pockets are present [cf. Fig. 2(c)]. Hansmann et al. [5] showed that under tensile strain and influence of correlation effects the $d_{z^{2}}$ states can be lifted above the Fermi level and fully depopulated [cf. Fig. 2(d)].

We begin our analysis of the $k$-resolved data by investigating the spectra taken with a photon energy of $710 \mathrm{eV}$, which corresponds to a $k$-space cut at $k_{z}=\frac{\pi}{c}$ (see Appendix B). Here the hole pockets should always be observable, independent of dimensionality or possible strain- or correlation-induced orbital polarization. Figures 3(a) and 3(c) show the energy distribution curves (EDCs) and Figs. 3(b) and 3(d) the corresponding momentum distribution curves (MDCs) of the $(4 / / 4)$ and $(2 / / 2)$ sample, respectively, along the $A-Z-A$ direction of the BZ. Strongly dispersive O $2 p$-derived bands are observed in both samples at lower energies between -8 and $-10 \mathrm{eV}$, indicating the high-quality crystalline structure of our SLs. Furthermore, the periodic band dispersion allows a clear identification of the high-symmetry points $\mathrm{Z}$ at $k_{\|}=0 \AA^{-1}$ and $\mathrm{A}$ at $k_{\|}=\frac{\pi}{a}$. The superposition of LAO and LNO states between -8 and $-2 \mathrm{eV}$ hinders the detection of dispersions of the $\mathrm{O} 2 p$-derived bands in this region. Near $E_{F}$ one can clearly identify the $\mathrm{Ni} 3 d$ states, although their intensity is much lower in comparison to the $\mathrm{O} 2 p$-derived valence states.

A more detailed view on the Ni $3 d$ states near the Fermi level is given in Figs. 4(a) and 4(b), where the EDCs taken in $\mathrm{A}-\mathrm{R}-\mathrm{A}$ direction are shown for both samples. Interestingly, a large, $k$-independent contribution to the intensity at the Fermi level is found. This loss of momentum information may be explained by nondirect transitions due to polaronic effects, as 

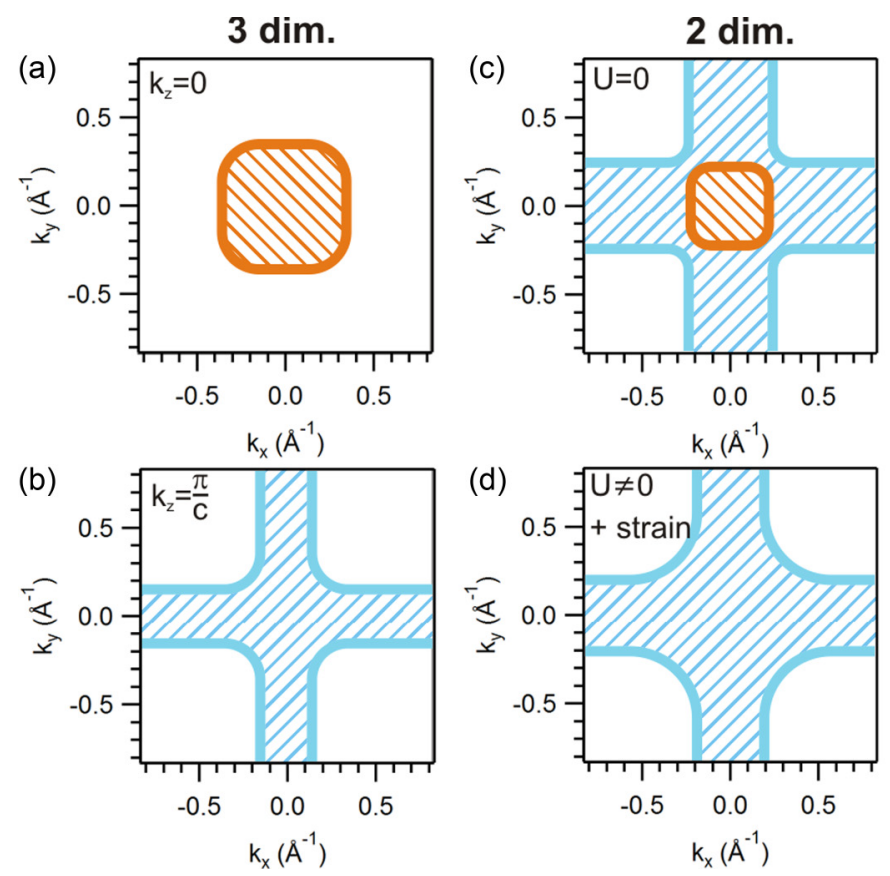

major orbital character:

Ni d $z^{2}$

Ni $\mathrm{d}_{\mathrm{x}^{2}-\mathrm{y}^{2}}$

(e)

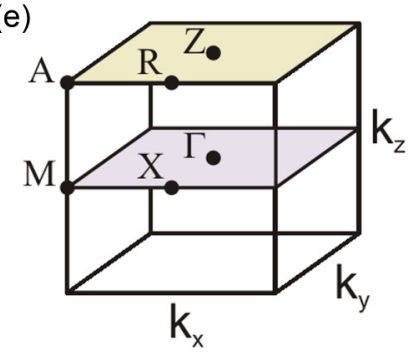

FIG. 2. (Color online) Schematic cuts (solid lines) through the 3D Fermi surface of bulk LNO at (a) $k_{z}=0 \AA^{-1}$ and (b) $k_{z}=\frac{\pi}{c}$ as well as the $k_{z}$-independent 2D FS (c) without and (d) including correlation effects (symbolized in the figure by zero and nonvanishing on-site Coulomb repulsion energy $U$ ) and tensile strain according to Refs. [39] and [5], respectively. The shaded areas indicate the occupied states. (e) Drawing of the cubic Brillouin zone including the high-symmetry points.

also reported for other oxide compounds [40,41]. Nevertheless, there is residual $k$-dependent intensity modulation at the Fermi level in both samples (though of different strength), consistent with the expected Fermi level crossing of the $d_{x^{2}-y^{2}}$ band along A-R-A (cf. Fig. 2). The EDCs of the (4//4) SL [see Fig. 4(a)] exhibit an intense structure around $\mathrm{R}$ which can be assigned to the occupied states between the hole pockets. However, while Eguchi et al. found at this high-symmetry point a clearly dispersive band in their SX-ARPES study of a thick LNO film under comparable experimental conditions [31], no dispersion is discernible in our measurement, already indicating a deviation of the SL from the bulk band structure.

In comparison to the $(4 / / 4) \mathrm{SL}$, where the $\mathrm{Ni} t_{2 g}$ and $e_{g}$ states are well separated in energy, both features are considerably smeared out in the $(2 / / 2)$ SL [see Fig. 4(b)]. Since the SL is well ordered as proven by the distinct dispersions in the $\mathrm{O} 2 p$ valence band, we attribute this behavior to the pronounced loss of QP coherence, as already discussed above. The reduced QP coherence furthermore prevents the identification of possible dispersive structures. An only very weak but significant intensity modulation around the $\mathrm{R}$ point may be interpreted as a remnant of the occupied $d_{x^{2}-y^{2}}$ band states sitting on top of a largely $k$-independent incoherent background.

\section{X-ray photoelectron diffraction}

Figures 5(a)-5(e) show angle-resolved photoelectron distribution maps measured at different fixed binding energies (constant-energy maps). The experimental geometry is sketched in Fig. 5(f), where the angles $\theta$ and $\phi$ are rotation angles around the sample axes as indicated and describe the direction of photoelectron emission with respect to the surface normal [34]. By adjusting $\theta$ and $\phi$ a 2D cut in momentum space, spanned by $\mathbf{k}_{\|}^{a}$ and $\mathbf{k}_{\|}^{b}$, can be sampled (in our experiments the sample orientation was such that the $k_{x}$ and $k_{y}$ axes, defining the first Brillouin zone as in Fig. 2, were rotated by $45^{\circ}$ with respect to $\mathbf{k}_{\|}^{a}$ and $\mathbf{k}_{\|}^{b}$ ). For the special case $\phi=0^{\circ}$, the relation between the initial state momentum component parallel to the sample surface, $k_{\|}^{a}$, and $\theta$ is given by the following equation:

$$
k_{\|}^{a}=\frac{\sqrt{2 m_{e}}}{\hbar} \sqrt{E_{\text {kin }}} \sin \theta-k_{\|}^{\mathrm{ph}},
$$

where $m_{e}$ is the free-electron mass and $E_{\text {kin }}$ the kinetic energy of the photoelectrons. $k_{\|}^{\mathrm{ph}}$ denotes the parallel component of the photon momentum $p^{\mathrm{ph}}=\hbar k^{\mathrm{ph}}=\frac{h v}{c}$, which is transferred to the emitted photoelectron [see Fig. 5(g)]. While this contribution can be neglected in low-energy ARPES measurements $(h v<100 \mathrm{eV})$, it has to be taken into account in SX-ARPES when converting the angular scale to momentum scale. Note that in the given geometry a correction only for the $\theta$ axis is sufficient, since the variation of $\phi \neq 0^{\circ}$ is small and thus the lateral contribution of the photon momentum is negligible.

Figures 5(a) and 5(b) show the constant-energy maps of the $(4 / / 4) \mathrm{SL}$ at the Fermi level $\left(e_{g}\right.$ states$)$ and at $E=-0.8 \mathrm{eV}$ ( $t_{2 g}$ states) integrated over energy windows of $\pm 0.15 \mathrm{eV}$ and $\pm 0.30 \mathrm{eV}$, respectively. The energy windows were chosen such that a satisfactorily low noise level in the intensity distribution maps was achieved. Note that only part of the full intensity range is captured by the color scale as is indicated for each map. Remarkable intensity modulations are observed 

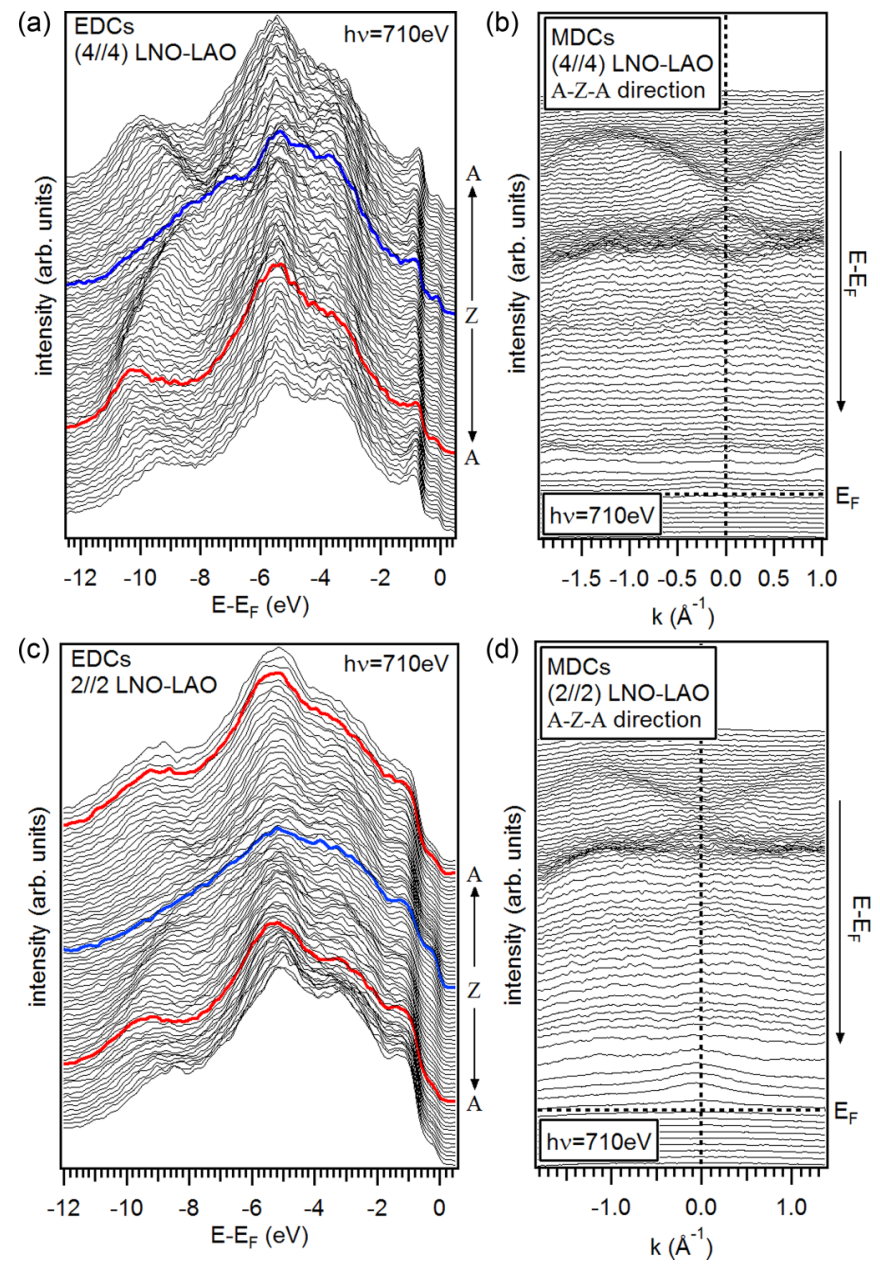

FIG. 3. (Color online) (a), (b) The energy and momentum distribution curves (EDCs and MDCs) of the (4//4) SL taken in the A-Z-A direction show at lower energies strongly dispersive $\mathrm{O} 2 p$-derived bands. (c), (d) These bands are also detectable in the EDCs and MDCs of the (2//2) SL measured in same direction. The observation of dispersive bands confirms the high crystalline quality of both SL and allows the identification of the high-symmetry points.

in the map of the $e_{g}$ states. A more detailed analysis may be obtained from the angle-dependent measurements of the $t_{2 g}$ states in Fig. 5(b): The chosen integration range corresponds roughly to the bandwidth of the fully occupied $t_{2 g}$ states, and hence the spectral weight distribution should simply reflect the momentum distribution function $n(\vec{k})$ which for a completely occupied band is constant $=1$. However, strong intensity modulations are clearly observable exhibiting a geometric symmetry with respect to $\theta=0^{\circ}$ [cf. Fig. 5(f)] but none with respect to any high-symmetry point or line in $k$ space. This suggests that most of the intensity modulation may be attributed to x-ray photoelectron diffraction (XPD) [42]. Here, the excited photoelectron is viewed as a spherical wave originating at some emitting atom and elastically scattered off the neighboring atoms in the lattice. Interference results in an intensity pattern which is symmetric in the angle coordinates while modulations due to the electronic structure are symmetric in momentum space. Note that for the high
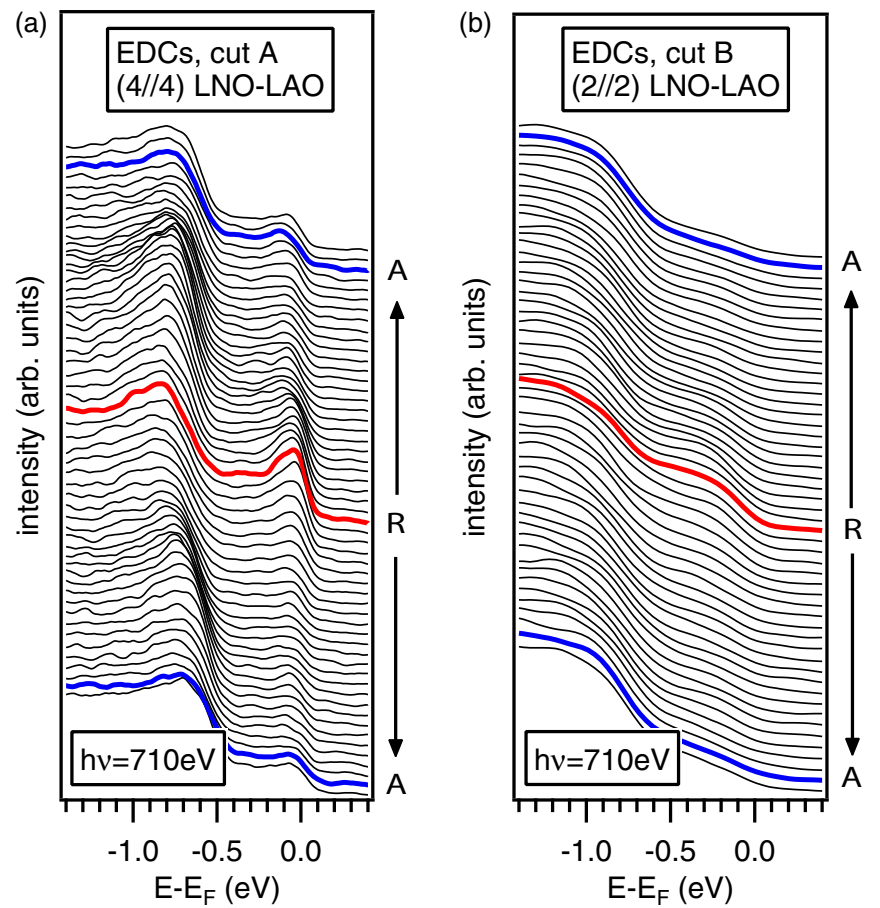

FIG. 4. (Color online) EDCs near $E_{F}$ measured along two cutslabeled $\mathrm{A}$ and $\mathrm{B}-$ in the $\mathrm{A}-\mathrm{R}-\mathrm{A}$ direction. The locations of both cuts are indicated in Fig. 6. (a) The EDCs of the (4//4) SL exhibit no dispersion in the hole pocket states around $\mathrm{R}$ within experimental resolution. (b) Both the Ni $t_{2 g}$ and $e_{g}$ states of the (2//2) SL are smeared out due to the loss of QP coherence. Only a residue of $k$-dependent intensity can be found near $\mathrm{R}$.

kinetic energies used here forward scattering dominates the XPD process [43]. The appearance of this effect in SXARPES measurements has been reported also for other oxide SL systems [44]. Since photoemission and photoelectron diffraction have to be considered as one quantum mechanical process, any correction for XPD effects necessarily relies on simplifying assumptions whose justification is difficult to predict. A heuristic procedure [44] to subtract out XPS features from the original data turned out to be not useful in the case of the LNO SLs studied here (not shown). Hence, we only display raw data throughout the paper.

The angular intensity distribution of the $e_{g}$ photoemission map is additionally affected by the only partial $k$-space occupation of the $e_{g}$ states, leading to the strongly reduced signal at small angles (with some remaining nonzero incoherent background). XPD effects are nonetheless well visible in the occupied part of the Brillouin zone, e.g., as pronounced intensity maxima at $\theta \approx 10^{\circ}$ (such are seen also in the $t_{2 g}$ maps).

The identification of XPD as origin of the intensity modulations in the Ni $3 d$ states is supported by angle-resolved data of the Ni 3 pcore level at $E=-67 \mathrm{eV}$ [see Fig. 5(c)]. The data were taken at a photon energy of $h v=780 \mathrm{eV}$ (corresponding to a total energy resolution of about $70 \mathrm{meV}$ ) in order to ensure the same kinetic energy, i.e., same wavelength, as in the ARPES maps in Figs. 5(a) and 5(b). The Ni 3p XPD pattern-since core levels are dispersionless, without 
(a)

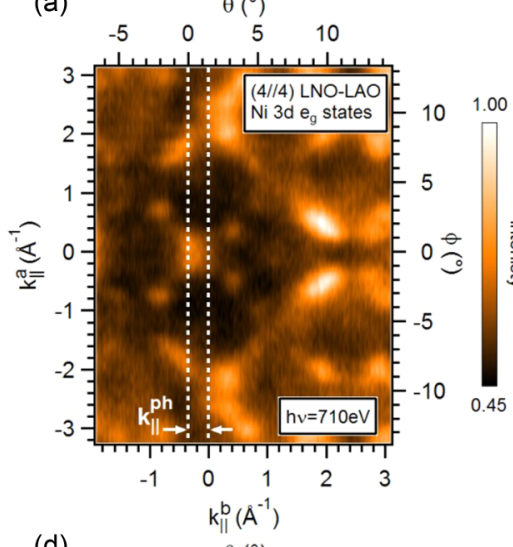

(d)

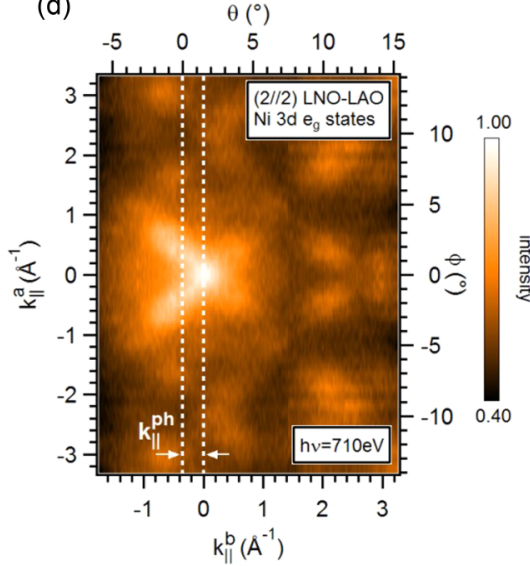

(b)

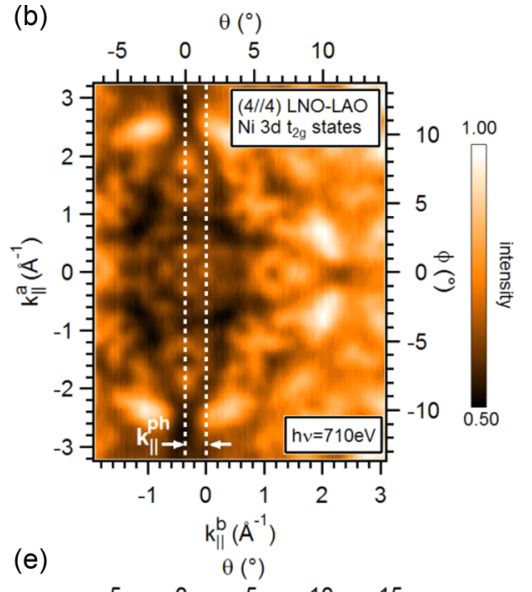

(c)

$(\mathrm{f})$

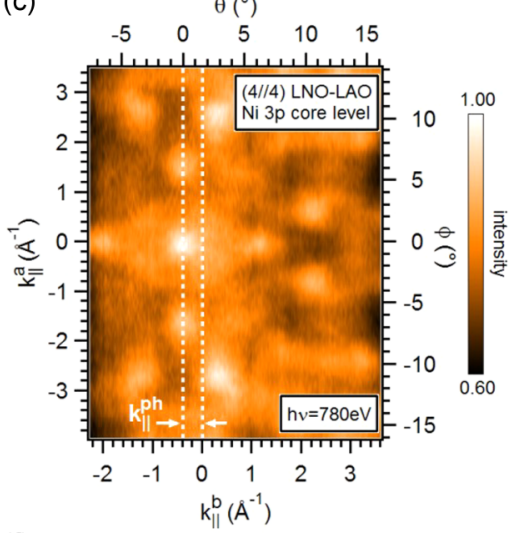

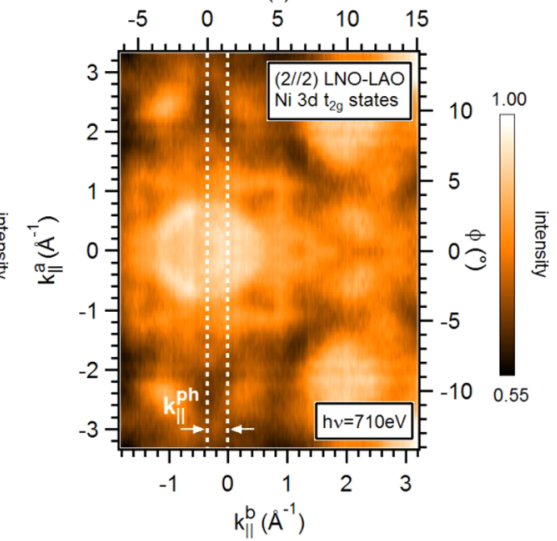

FIG. 5. (Color online) Angle-resolved constant-energy maps of the (4//4) and (2//2) SL measured at different binding energies. Only part of the full intensity range is captured by the color scale as is indicated for each map. (a) The map of the (4//4) SL at $E_{F}\left(e_{g}\right.$ states) exhibits strong intensity modulation. (b) The intensity map measured at $-0.8 \mathrm{eV}$ ( $t_{2 g}$ states) displays symmetry with respect to $\theta=0^{\circ}$. Thus, the observed modulations may be identified as a result of photoelectron diffraction. (c) Angle-resolved measurements of the Ni $3 p$ core level, taken at a similar kinetic energy as the valence band maps in (a) and (b), show a comparable pattern. (d), (e) The angle-resolved data of the $e_{g}$ (d) and $t_{2 g}$ states (e) taken on the (2//2) SL provide similar results. It should be noted that the figures of the (2//2) SL were made by mirroring the data at $\phi=0^{\circ}$, which does not reduce the information on the FS, since the line $\phi=0^{\circ}$ corresponds to a real mirror plane of the experiment (in contrast to the line $\theta=0^{\circ}$, which explains the systematic intensity asymmetry in the $\theta$ direction due to matrix-element effects). (f) Schematic view of the experimental geometry. (g) The projection of the soft-x-ray photon momentum parallel and perpendicular to the sample surface at arbitrary $\theta$ and $\phi=0^{\circ}$.

intensity modulations owing to a momentum dependence of the electronic structure-shows clear structure-induced symmetries about $\theta=0^{\circ}$ and $\phi=0^{\circ}$ and contains many of the features also seen in the $\mathrm{Ni} 3 d$ maps.

We observe similar intensity distributions in the angleresolved maps of the $e_{g}$ and $t_{2 g}$ states of the $(2 / / 2) \mathrm{SL}$, as shown in Figs. 5(d) and 5(e), respectively. While the map of the fully occupied $t_{2 g}$ band again exhibits clear XPD-induced symmetry with respect to $\theta=0^{\circ}$, the map of the $e_{g}$ states displays a superposition of $k$-dependent state occupancy, e.g., the X-shaped structure at $k_{\|}^{a, b}=0 \AA^{-1}$, and XPD-induced modulations, e.g., at $\theta \approx 10^{\circ}$.

The difference in the observed intensity modulations between both SLs at $E_{F}$ is explained on the one hand by the $k$-dependent occupancy of the Ni $e_{g}$ states (see discussion in the next section). On the other hand the different layer thicknesses of LAO and LNO may lead to slightly different XPD modulation patterns, especially in the angle-resolved intensity distribution maps of the $t_{2 g}$ states.

\section{Fermi surfaces}

After having identified the angular XPD effects in the photoemission maps taken at $E_{F}$ ( $e_{g}$ states $)$ we now turn to the underlying $k$-dependent structures resulting from the electronic structure, i.e., the Fermi surface of the buried LNO layers. For better identification we compare the measured intensity distributions, provided with a momentum scale, with the calculated Fermi surfaces in Fig. 6.

Starting with the (4//4) SL [Fig. 6(a)], recorded at $h v=$ $710 \mathrm{eV}$, which in the free-electron approximation for the final states corresponds to $k_{z}=\frac{\pi}{c}$, we find qualitatively very good agreement with the calculated $k$-space occupation at this very $k_{z}$ (gray shaded schematic). In particular, the hole pockets around the A points are clearly visible. As already discussed above, due to the moderate energy and momentum resolution no sharp Fermi-level crossings of the only very weakly dispersing peaks (cf. Figs. 3 and 4) can be observed. With the energy integration range $( \pm 0.15 \mathrm{eV})$ being comparable to the $e_{g}$ bandwidth these maps essentially reflect the momentum 

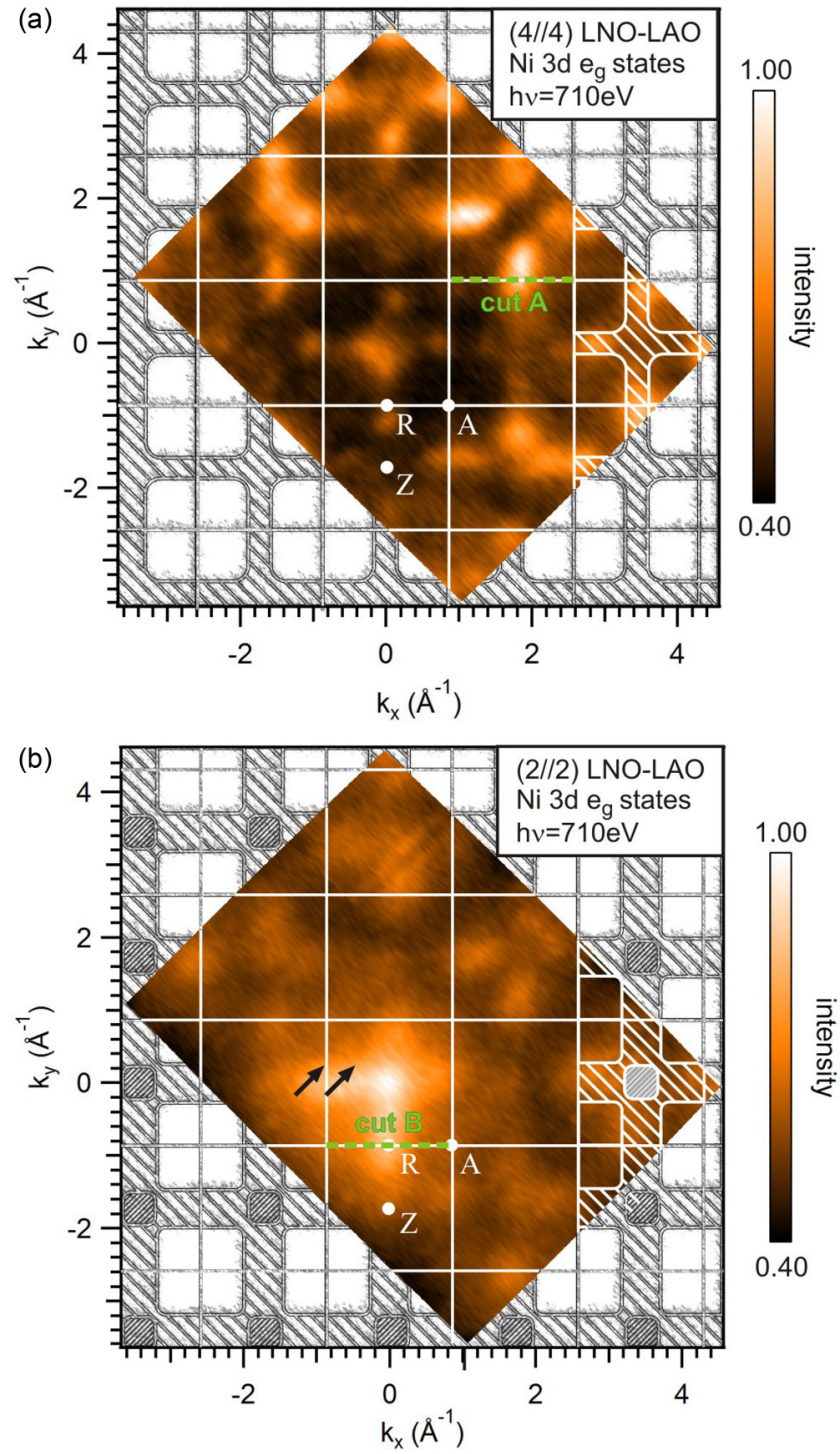

FIG. 6. (Color online) (a) The $k$-resolved intensity distribution map of the $(4 / / 4) \mathrm{SL}$ taken at $E_{F}$ with $k_{z}=\frac{\pi}{c}(710 \mathrm{eV})$ including several BZs shows the distinct hole pockets around the A points, in line with band theory (shaded drawings). (b) The $k$-resolved map of the $(2 / / 2) \mathrm{SL}$ is in good agreement with the $k_{z}$-independent 2D FS with hole pockets around the A points and an additional electron pocket around the $\mathrm{Z}$ point. The latter one is only clearly observable at $k_{x, y}=0 \AA^{-1}$, presumably due to matrix element effects. The arrows indicate a possible nesting vector of the FS, which agrees with the SDW wave vector $\mathbf{Q}_{\mathrm{SDW}}=2 \pi\left(\frac{1}{4}, \frac{1}{4}, 0\right)$. The lines $\mathrm{A}$ and B denote the $k$-space cuts probed by the EDCs in Fig. 4 . The intensity of both maps is normalized to that at $k_{x, y}=0 \AA^{-1}$.

distribution function $n(\vec{k})$, i.e., the occupied $k$ space between the hole pockets rather than the FS contours [cf. shaded areas in Fig. 6(a)] [45].

The Fermi level intensity map of the $(2 / / 2)$ SL shown in Fig. 6(b) basically resembles the expected momentum distribution function in the $2 \mathrm{D}$ limit. Compared to the data of the $(4 / / 4)$ SL the structures are broader in $k$ space. This can be explained by the change of the FS from the 3D to the 2D topology, where the hole pockets have to shrink in order to keep the Fermi volume constant according to the Luttinger theorem (cf. Fig. 2), resulting in a broader occupied $k$-space region between the hole pockets. At the $\mathrm{Z}$ point of the first $\mathrm{BZ}$ of the $(2 / / 2)$ SL some additional intensity is clearly visible, but much less so in higher-order zones, possibly due to matrix element effects. It is tempting to assign this intensity to the additional electron pocket in the center of the BZ predicted for the 2D limit of Fig. 2(c). That this intensity maximum is clearly centered around $k_{\|}^{x, y}=0 \AA^{-1}$ [and not $\theta=0^{\circ}$; see Fig. 5(d)] supports the interpretation as a genuine part of the band structure and rules out an XPD effect.

Both maps are taken at the same photon energy corresponding to $k_{z}=\frac{\pi}{c}$. Since for a 3D, i.e., bulklike, FS the electron pocket should only be visible at the $\Gamma$ points (corresponding to integer multiples of $\frac{2 \pi}{c}$ in the $k_{z}$ direction within the extended zone scheme), as can be seen from the theoretical FS in Fig. 2, it is interesting to look at FS cuts containing a $\Gamma$ point. The next available $\Gamma$ point requires a photon energy of $h v=630 \mathrm{eV}$ (see Appendix B). The intensity distribution map of the $(2 / / 2) \mathrm{SL}$ shown in Fig. 7(a) indeed exhibits significant intensity at the $\Gamma$ point, which may be a result of overlapping intensity derived from the Ni $d_{z^{2}}$ states at $\Gamma$ and XPD-induced modulations at $\theta=0^{\circ}$. The diffuse intensity distribution towards the BZ edge may be interpreted as residual spectral weight of the occupied states between the hole pockets, although the very low Fermi level intensity prevents a more detailed analysis. In any case, the ubiquity of the zone center intensity for all photon energies (and thus its $k_{z}$ independence) strongly supports the 2D character of the FS in the $(2 / / 2) \mathrm{SL}$.

Figure 7(b) shows the Fermi surface maps taken on the $(4 / / 4) \mathrm{SL}$ at $h v=630 \mathrm{eV}$. Due to the thicker LNO layer one would expect to slowly recover bulk behavior with a stronger electron pocket signal at the $\Gamma$ point. However, we found no evidence for a $k$-dependent state occupancy in our data. Rather, all intensity modulations exhibit a symmetry with respect to $\theta=0^{\circ}$, identifying them as XPD-induced structures. To verify this result, we additionally performed angle-resolved measurements on the $(10 / / 4) \mathrm{SL}$ at the same photon energy, since at 10 uc LNO layer thickness a closer approach to a fully established 3D FS can be expected [10]. However, also in this SL no significant $k$-dependent structures are detected; in particular, no electron pocket is found at the $\Gamma$ point [see Fig. 7(c)]. Furthermore, a $k$-space scan in the $k_{z}$ direction by varying the photon energy from 580 to $820 \mathrm{eV}$ (not shown here) did not reveal any signal which could be assigned to the Ni $d_{z^{2}}$ pocket around $k_{\|}^{a, b}=0 \AA^{-1}$.

\section{DISCUSSION}

The SX-ARPES data taken at $k_{z}=\frac{\pi}{c}(h v=710 \mathrm{eV})$ provide direct spectroscopic evidence of the predicted $\mathrm{Ni}$ $d_{x^{2}-y^{2}}$-derived hole pockets in all investigated SLs. However, for the electron pocket formed by $\mathrm{Ni} d_{z^{2}}$ states in the BZ center indications are only found in the $(2 / / 2)$ SL. The angle-resolved data of both the (4//4) SL and (10//4) SL taken in the central plane of the $\mathrm{BZ}\left(k_{z}=0 \AA^{-1}\right.$, i.e., $\left.h v=630 \mathrm{eV}\right)$ are strongly affected by XPD-induced intensity modulations and display no clear $k$-dependent state occupancy. This is a surprising result, because a recent SX-ARPES study on thick LNO films 

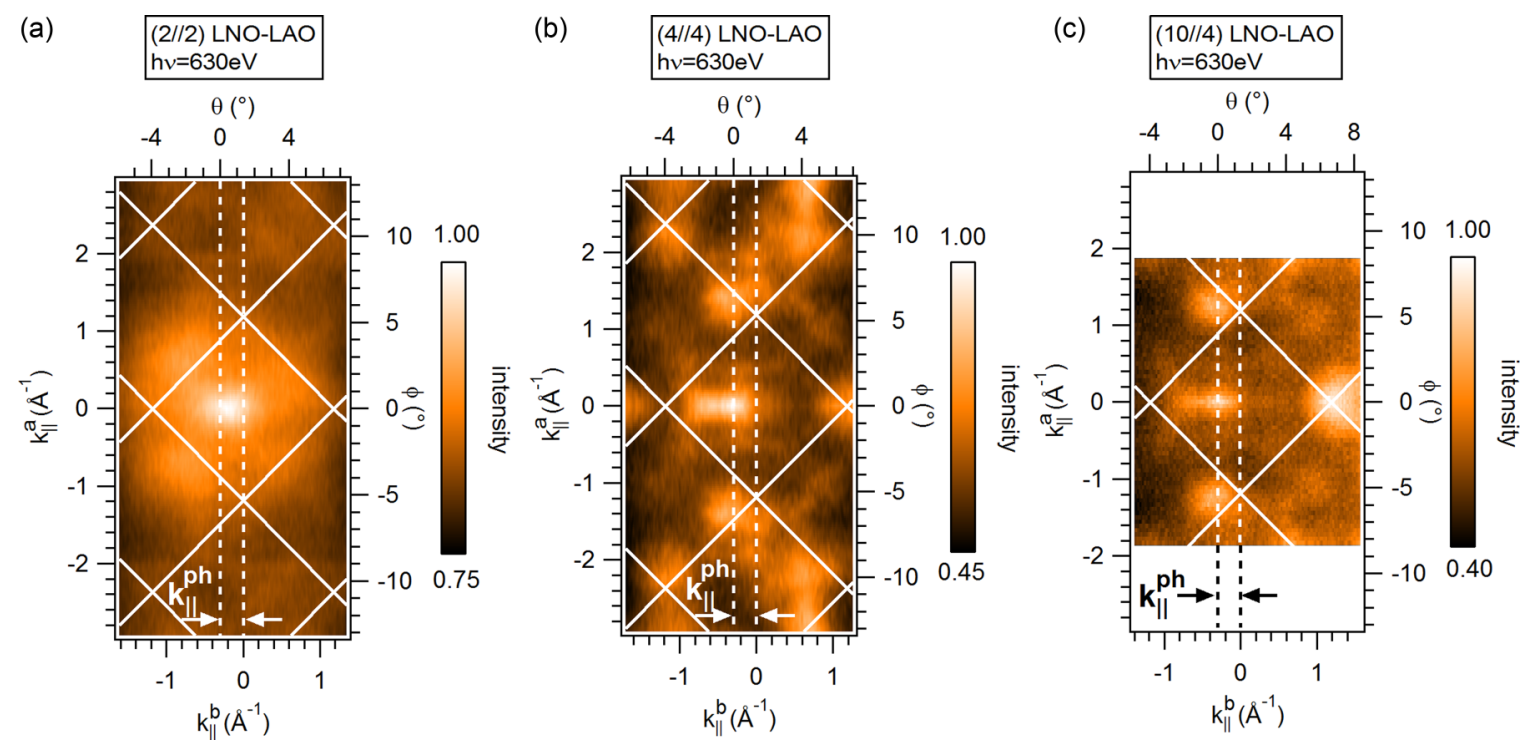

FIG. 7. (Color online) The angle-resolved intensity distribution maps of different LNO/LAO SL at $E_{F}$, measured at $h v=630 \mathrm{eV}$, which corresponds to $k_{z}=0 \AA^{-1}$. The white lines denote the BZ borders. (a) The data taken on the $(2 / / 2) \mathrm{SL}$ shows intensity at $k_{\|}^{a, b}=0 \AA^{-1}$, which may be assigned to the electron pocket, slightly interfering with the XPD-induced intensity maximum at $\theta=0^{\circ}$. The measured intensity distribution maps of the (b) (4//4) SL and (c) (10//4) SL are dominated by XPD effects. No electron pocket can be identified.

has reported clear evidence of the $\mathrm{Ni} d_{z^{2}}$ states and their 3D dispersion [31].

It may be tempting to attribute this difference between confined LNO layers in SLs and bulk LNO to the strain induced by the substrate. However, a recent resonant reflectometry study focusing on the correlation between strain and orbital polarization in LNO/LAO SLs provides evidence that compressive strain like in our samples enhances the $\mathrm{Ni} d_{z^{2}}$ band filling compared to the situation in unstrained LNO [11,18] while, conversely, tensile strain energetically lifts the $d_{z^{2}}$ orbital and causes its depopulation. This trend is supported by DFT calculations for ultrathin LNO films [46] and superlattices $[5,6,47,48]$. Thus, the scenario that compressive strain induced by the LSAO substrate is the reason for the strongly reduced $\mathrm{Ni} d_{z^{2}}$ occupancy in our samples can most likely be excluded.

Another explanation is a much stronger XPD effect in the SL samples compared to bare LNO films. The data of both the (4//4) SL and the (10//4) SL in Figs. 7(b) and 7(c), respectively, show nearly identical intensity patterns, despite their different LNO layer thickness. They share however the same thickness of the LAO capping layer, and therefore it seems likely that most of the detected intensity modulations are caused by photoelectron diffraction within that layer. In SXARPES of bulk LNO films most of the Ni $3 d$ signal will originate from the top surface layers, whereas in LAO-capped SLs the photoelectron wave excited from the buried LNO layers will have to pass through the LAO overlayer and thus be subject to additional scattering and interference effects. The stronger XPD intensity modulations may then distort or even obscure any underlying $k$-dependent band structure information. A test of this hypothesis would require a more systematic study of SL samples with varying LAO capping layer thickness.

A very clear and unambiguous result of the angle-integrated data in Fig. 1(b) is the change in the microscopic electronic structure from the $(4 / / 4)$ SL to the $(2 / / 2)$ SL, indicating a significant loss of QP coherence concomitant with the dimension-controlled MI crossover observed in transport. Different mechanisms have been proposed to explain the latter. For ultrathin LNO films 2D Anderson localization has been discussed [8], while other studies performed on ultrathin films [13] and SLs [23], both under tensile strain, attribute the insulating phase to charge disproportionation in the 2D ground state. Recent theoretical studies found strong evidence for a magnetic instability, i.e., the formation of a SDW, driven by FS nesting, with a wave vector $\mathbf{Q}_{\mathrm{SDW}}=2 \pi\left(\frac{1}{4}, \frac{1}{4}, \frac{1}{4}\right)$ as determined from the theoretical susceptibility $[20,21]$. This scenario is supported by spectroscopic data on $\mathrm{PrNiO}_{3}-\mathrm{PrAlO}_{3}$ superlattices [49]. Experimentally, muon-spin-rotation [12] and resonant x-ray diffraction experiments [17] indeed observed antiferromagnetic ordering at the predicted wave vector, but only in the $(2 / / 2)$ SL, independent of the used substrate, i.e., the induced strain. SLs with thicker LNO layers exhibit paramagnetic behavior, indicating that the SDW is closely linked to the reduced dimensionality.

Our measured spectroscopic data, especially on the FS topology, provide further support for the SDW scenario. Despite the pronounced loss of coherent quasiparticle weight in the $(2 / / 2) \mathrm{SL}$, the residual intensity modulation in the momentum distribution function $n(\vec{k})$ reproduces a nearly quadratic shape of the FS hole pockets, with flat contours providing a good basis for strong FS nesting. Within experimental resolution the wave vector predicted for the ideal $2 \mathrm{D}$ case $\left[\mathbf{Q}_{\mathrm{SDW}}=2 \pi\left(\frac{1}{4}, \frac{1}{4}, 0\right)\right]$ is indeed compatible with nesting of the experimental FS [see arrows in Fig. 6(b)]. On the other hand, it may seem surprising that FS signal can still be observed in our low-temperature data, i.e., well within the SDW phase, because a nesting instability would normally open a gap and destroy the FS. However, as already seen in the EDCs of the $(2 / / 2) \mathrm{SL}$, there is no full gap opening at $E_{F}$, at least within 
our experimental resolution. This behavior could be attributed to an insufficient SDW stabilization due to pronounced order parameter fluctuations, not fully unexpected in the 2D limit. This picture is supported by very recent magnetoresistivity measurements performed on similar LNO/LAO SLs [30]. Hepting et al. [49] also find that the SDW state remains metallic; i.e., the gap does not encompass the entire FS. Order parameter fluctuations may also explain the suppression of quasiparticle coherence and the absence of SDW-induced band backfolding, in remarkable contrast to recent ARPES results on ultrathin films [10].

\section{CONCLUSIONS}

In summary, we have investigated the electronic structure of compressively strained LNO/LAO SLs grown on LSAO substrates by angle-resolved soft-X-ray photoemission. $k$ integrated valence band spectra show a loss of quasiparticle coherence below 3 uc LNO layer thickness. Corresponding transport measurements exhibit a temperature-dependent MI crossover in the $(2 / / 2)$ SL, while the $(4 / / 4)$ and $(10 / / 4)$ SLs stay metallic down to low temperatures. While in thin films similar observations have been reported the microscopic mechanisms in thin films and superlattice structures might be diverse due to the different strain fields.

Although the analysis of the angle-resolved measurements is complicated by strong XPD-induced intensity modulations, the measured angle-resolved maps reveal a dimensional crossover of the FS from 3D in the (4//4) SL to 2D behavior in the $(2 / / 2)$ SL. By comparing the maps with results from DFT calculations, the Ni $d_{x^{2}-y^{2}}$ states, which form the hole states around the A points, are clearly identified in the FS of all measured SLs. Evidence for the electron pocket derived from Ni $d_{z^{2}}$ orbital is found only in the $(2 / / 2)$ SL, but could not be observed for SLs with thicker LNO and LAO layers. We attribute this to pronounced XPD effects in the LAO overlayer, which interfere with or even obscure $k$-dependent Fermi surface information.

The measured FS topology, in particular the shape of hole pockets in the $(2 / / 2) \mathrm{SL}$, support FS nesting. This is consistent with the scenario of a dimensionality-induced SDW instability, with the nesting properties of our experimental FS in excellent agreement with the reported SDW wave vector. Thus, our results strongly support magnetic ordering in the 2D ground state of ultrathin LNO layers embedded in a SL. In line with other experimental as well as theoretical studies, no indication of correlation-induced FS modifications was found for the SLs under compressive strain. Further studies, particularly on LNO/LAO SLs under tensile strain, are needed to investigate the tunability of the electronic structure by strain and correlation effects in the 2D limit.

\section{ACKNOWLEDGMENTS}

We thank C. S. Fadley and A. X. Gray for fruitful discussions. This work was supported by the Deutsche Forschungsgemeinschaft (FOR 1162 and TRR80, project G1) and the German Federal Ministry for Education and Research (05K10WW1). The results of this study benefited

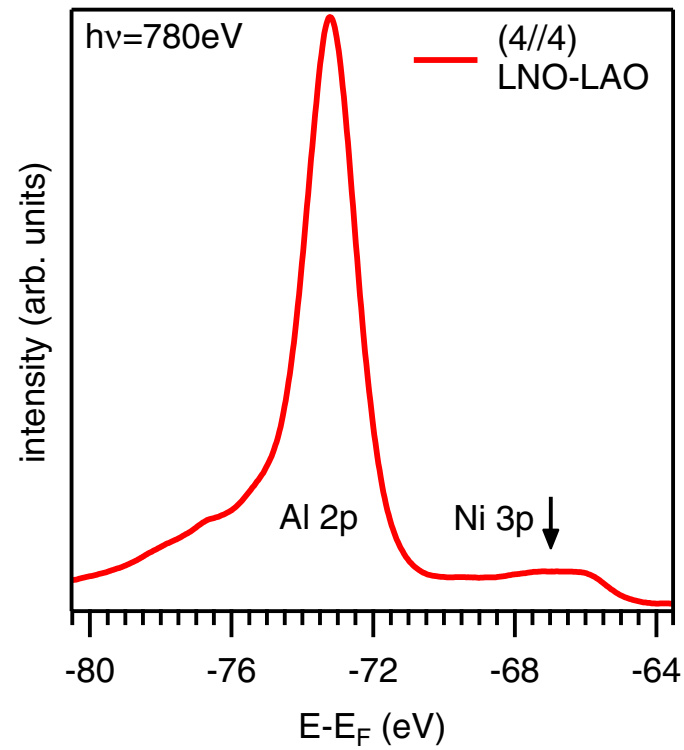

FIG. 8. (Color online) Core-level spectrum including the Al $2 p$ and Ni $3 p$ line taken at $h v=780 \mathrm{eV}$. From the position of the Al $2 p$ line the valence band offset of the LAO overlayer is determined. The arrow indicates the binding energy used for the angle-resolved core-level map in Fig. 5(c).

strongly from pilot measurements performed at SPring-8, Japan (2010B1339).

\section{APPENDIX A: VALENCE BAND OFFSET OF THE $\mathrm{LaAlO}_{3}$ OVERLAYER $^{2}$}

In the SLs the LNO layer is buried below a LAO capping layer. Thus, the measured valence band is a superposition of the LAO and LNO valence states. Only, if the valence band maximum of the insulating $\mathrm{LAO}, E_{\mathrm{VBM}}^{\mathrm{LNO} / \mathrm{LAO}}$, is far enough below the Fermi level $E_{F}$, the observed spectral weight near $E_{F}$ can unambiguously be assigned to the Ni $3 d$ states in the LNO layers. $E_{\mathrm{VBM}}^{\mathrm{LNO} / \mathrm{LAO}}$ is determined by analyzing the position of a suitable core level from LAO, e.g., Al $2 p$, and comparing its position with a reference system containing LAO, where core-level position and valence band onset are well known. Here, we use as reference the well-studied $\mathrm{LaAlO}_{3} / \mathrm{SrTiO}_{3}$ heterostructure with $E_{\mathrm{A} 12 p}^{\mathrm{LAO} / \mathrm{STO}} \approx-74.35 \mathrm{eV}$ and $E_{\mathrm{VBM}}^{\mathrm{LAO} / \mathrm{STO}} \approx$ $-3.1 \mathrm{eV}$ [50]. The energy position of the $\mathrm{Al} 2 p$ core level in the LNO/LAO SLs is found to be $E_{\mathrm{Al} 2 p}=-73.20 \mathrm{eV}$ (see Fig. 8), independent of the LAO and LNO layer thickness. From the obvious relationship

$$
E_{\mathrm{VBM}}^{\mathrm{LNO} / \mathrm{LAO}}=E_{\mathrm{Al} 2 p}^{\mathrm{LNO} / \mathrm{LAO}}-E_{\mathrm{Al} 2 p}^{\mathrm{LAO} / \mathrm{STO}}+E_{\mathrm{VBM}}^{\mathrm{LAO} / \mathrm{STO}}
$$

we determine the position of the valence band maximum in the LAO overlayer as $E_{\mathrm{VBM}}^{\mathrm{LNO} / \mathrm{LAO}}=-1.95 \pm 0.1 \mathrm{eV}$. Consequently, any spectral weight measured between $E_{F}$ and $\approx-2 \mathrm{eV}$ can only result from the $\mathrm{Ni} 3 d$ states in the LNO layers.

\section{APPENDIX B: $k_{z}$ DEPENDENCE FOR BULK $\mathrm{LaNiO}_{3}$}

The relationship between the kinetic energy of the photoelectrons and the momentum perpendicular to the sample surface is described by the following equation including 
the nonnegligible photon momentum component $k_{\perp}^{\mathrm{ph}}$ [see Fig. 5(g)]:

$$
k_{z}=\sqrt{2 m_{e} / \hbar^{2}}\left(V_{0}+E_{k} \cos ^{2} \theta\right)^{1 / 2}-k_{\perp}^{\mathrm{ph}},
$$

where $m_{e}$ is the free-electron mass, $V_{0}$ the inner potential, $E_{k}$ the kinetic energy, and $\theta$ the emission angle. For thick
LNO films a value of $V_{0}=10 \mathrm{eV}$ has been reported [31]. We do not expect a large variation of this phenomenological parameter for our LNO/LAO SLs. Thus, by using the lattice constant perpendicular to the surface $c=3.840 \AA$ [18] and taking the experimental geometry into account, the photon energies required for probing Fermi level states at the center $\left(k_{z}=0 \AA^{-1}\right)$ and the edge of the $\mathrm{BZ}\left(k_{z}=\frac{\pi}{c}\right)$ perpendicular to the surface are $h v=630 \mathrm{eV}$ and $h v=710 \mathrm{eV}$, respectively.
[1] H. Y. Hwang, Y. Iwasa, M. Kawasaki, B. Keimer, N. Nagaosa, and Y. Tokura, Nat. Mater. 11, 103 (2012).

[2] J. B. Torrance, P. Lacorre, A. I. Nazzal, E. J. Ansaldo, and C. Niedermayer, Phys. Rev. B 45, 8209 (1992).

[3] M. Imada, A. Fujimori, and Y. Tokura, Rev. Mod. Phys. 70, 1039 (1998).

[4] J. Chaloupka and G. Khaliullin, Phys. Rev. Lett. 100, 016404 (2008).

[5] P. Hansmann, X. Yang, A. Toschi, G. Khaliullin, O. K. Andersen, and K. Held, Phys. Rev. Lett. 103, 016401 (2009).

[6] P. Hansmann, A. Toschi, X. Yang, O. K. Andersen, and K. Held, Phys. Rev. B 82, 235123 (2010).

[7] R. Scherwitzl, P. Zubko, C. Lichtensteiger, and J.-M. Triscone, Appl. Phys. Lett. 95, 222114 (2009).

[8] R. Scherwitzl, S. Gariglio, M. Gabay, P. Zubko, M. Gibert, and J.-M. Triscone, Phys. Rev. Lett. 106, 246403 (2011).

[9] E. Sakai, M. Tamamitsu, K. Yoshimatsu, S. Okamoto, K. Horiba, M. Oshima, and H. Kumigashira, Phys. Rev. B 87, 075132 (2013).

[10] H. K. Yoo, S. I. Hyun, L. Moreschini, H.-D. Kim, Y. J. Chang, C. H. S. N. D. W. Jeong, S. Sinn, Y. S. Kim, A. Bostwick, E. Rotenberg, J. H. Shim, and T. W. Noh, Sci. Rep. 5, 8746 (2015).

[11] E. Benckiser, M. W. Haverkort, S. Brück, E. Goering, S. Macke, A. Frañó, X. Yang, O. K. Andersen, G. Cristiani, H.-U. Habermeier, A. V. Boris, I. Zegkinoglou, P. Wochner, H.-J. Kim, V. Hinkov, and B. Keimer, Nat. Mater. 10, 189 (2011).

[12] A. V. Boris, Y. Matiks, E. Benckiser, A. Frano, P. Popovich, V. Hinkov, P. Wochner, M. Castro-Colin, E. Detemple, V. K. Malik, C. Bernhard, T. Prokscha, A. Suter, Z. Salman, E. Morenzoni, G. Cristiani, H.-U. Habermeier, and B. Keimer, Science 332, 937 (2011).

[13] J. Chakhalian, J. M. Rondinelli, J. Liu, B. A. Gray, M. Kareev, E. J. Moon, N. Prasai, J. L. Cohn, M. Varela, I. C. Tung, M. J. Bedzyk, S. G. Altendorf, F. Strigari, B. Dabrowski, L. H. Tjeng, P. J. Ryan, and J. W. Freeland, Phys. Rev. Lett. 107, 116805 (2011)

[14] A. M. Kaiser, A. X. Gray, G. Conti, J. Son, A. Greer, A. Perona, A. Rattanachata, A. Y. Saw, A. Bostwick, S. Yang, S.-H. Yang, E. M. Gullikson, J. B. Kortright, S. Stemmer, and C. S. Fadley, Phys. Rev. Lett. 107, 116402 (2011).

[15] A. X. Gray, A. Janotti, J. Son, J. M. LeBeau, S. Ueda, Y. Yamashita, K. Kobayashi, A. M. Kaiser, R. Sutarto, H. Wadati, G. A. Sawatzky, C. G. Van de Walle, S. Stemmer, and C. S. Fadley, Phys. Rev. B 84, 075104 (2011).

[16] J. W. Freeland, J. Liu, M. Kareev, B. Gray, J. W. Kim, P. Ryan, R. Pentcheva, and J. Chakhalian, Europhys. Lett. 96, 57004 (2011).

[17] A. Frano, E. Schierle, M. W. Haverkort, Y. Lu, M. Wu, S. BlancoCanosa, U. Nwankwo, A. V. Boris, P. Wochner, G. Cristiani,
H. U. Habermeier, G. Logvenov, V. Hinkov, E. Benckiser, E. Weschke, and B. Keimer, Phys. Rev. Lett. 111, 106804 (2013).

[18] M. Wu, E. Benckiser, M. W. Haverkort, A. Frano, Y. Lu, U. Nwankwo, S. Brück, P. Audehm, E. Goering, S. Macke, V. Hinkov, P. Wochner, G. Christiani, S. Heinze, G. Logvenov, H.-U. Habermeier, and B. Keimer, Phys. Rev. B 88, 125124 (2013).

[19] M. J. Han, X. Wang, C. A. Marianetti, and A. J. Millis, Phys. Rev. Lett. 107, 206804 (2011).

[20] S. B. Lee, R. Chen, and L. Balents, Phys. Rev. Lett. 106, 016405 (2011).

[21] S. B. Lee, R. Chen, and L. Balents, Phys. Rev. B 84, 165119 (2011).

[22] A. S. Disa, F. J. Walker, S. Ismail-Beigi, and C. H. Ahn, APL Materials 3, 062303 (2015).

[23] J. Liu, S. Okamoto, M. van Veenendaal, M. Kareev, B. Gray, P. Ryan, J. W. Freeland, and J. Chakhalian, Phys. Rev. B 83, 161102 (2011).

[24] J. Hwang, J. Son, J. Y. Zhang, A. Janotti, C. G. Van de Walle, and S. Stemmer, Phys. Rev. B 87, 060101 (2013).

[25] S. J. May, J.-W. Kim, J. M. Rondinelli, E. Karapetrova, N. A. Spaldin, A. Bhattacharya, and P. J. Ryan, Phys. Rev. B 82, 014110 (2010).

[26] S. J. May, C. R. Smith, J.-W. Kim, E. Karapetrova, A. Bhattacharya, and P. J. Ryan, Phys. Rev. B 83, 153411 (2011).

[27] H. Qi, M. Kinyanjui, J. Biskupek, D. Geiger, E. Benckiser, H.-U. Habermeier, B. Keimer, and U. Kaiser, J. Mater. Sci. 50, 5300 (2015).

[28] M. K. Kinyanjui, Y. Lu, N. Gauquelin, M. Wu, A. Frano, P. Wochner, M. Reehuis, G. Christiani, G. Logvenov, H.-U. Habermeier, G. A. Botton, U. Kaiser, B. Keimer, and E. Benckiser, Appl. Phys. Lett. 104, 221909 (2014).

[29] J. Hwang, J. Y. Zhang, J. Son, and S. Stemmer, Appl. Phys. Lett. 100, 191909 (2012).

[30] D. P. Kumah, A. S. Disa, J. H. Ngai, H. Chen, A. Malashevich, J. W. Reiner, S. Ismail-Beigi, F. J. Walker, and C. H. Ahn, Adv. Mater. 26, 1935 (2014).

[31] R. Eguchi, A. Chainani, M. Taguchi, M. Matsunami, Y. Ishida, K. Horiba, Y. Senba, H. Ohashi, and S. Shin, Phys. Rev. B 79, 115122 (2009).

[32] P. D. C. King, I. H. Wei, F. Y. Nie, M. Uchida, C. Adamo, S. Zhu, X. He, I. Bozovic, D. G. Schlom, and M. K. Shen, Nat. Nanotech. 9, 443 (2014).

[33] G. Berner, M. Sing, H. Fujiwara, A. Yasui, Y. Saitoh, A. Yamasaki, Y. Nishitani, A. Sekiyama, N. Pavlenko, T. Kopp, C. Richter, J. Mannhart, S. Suga, and R. Claessen, Phys. Rev. Lett. 110, 247601 (2013). 
[34] V. N. Strocov, X. Wang, M. Shi, M. Kobayashi, J. Krempasky, C. Hess, T. Schmitt, and L. Patthey, J. Synchrotron Radiat. 21, 32 (2014).

[35] J. L. García-Muñoz, J. Rodríguez-Carvajal, P. Lacorre, and J. B. Torrance, Phys. Rev. B 46, 4414 (1992).

[36] A. Frano, E. Benckiser, Y. Lu, M. Wu, M. Castro-Colin, M. Reehuis, A. V. Boris, E. Detemple, W. Sigle, P. van Aken, G. Cristiani, G. Logvenov, H.-U. Habermeier, P. Wochner, B. Keimer, and V. Hinkov, Adv. Mater. 26, 258 (2014).

[37] V. N. Strocov, T. Schmitt, U. Flechsig, T. Schmidt, A. Imhof, Q. Chen, J. Raabe, R. Betemps, D. Zimoch, J. Krempasky, X. Wang, M. Grioni, A. Piazzalunga, and L. Patthey, J. Synchrotron Radiat. 17, 631 (2010).

[38] K. Horiba, R. Eguchi, M. Taguchi, A. Chainani, A. Kikkawa, Y. Senba, H. Ohashi, and S. Shin, Phys. Rev. B 76, 155104 (2007).

[39] N. Hamada, J. Phys. Chem. Solids 54, 1157 (1993).

[40] D. S. Dessau, T. Saitoh, C.-H. Park, Z.-X. Shen, P. Villella, N. Hamada, Y. Moritomo, and Y. Tokura, Phys. Rev. Lett. 81, 192 (1998).

[41] D. Schrupp, M. Sing, M. Tsunekawa, H. Fujiwara, S. Kasai, A. Sekiyama, S. Suga, T. Muro, V. A. M. Brabers, and R. Claessen, Europhys. Lett. 70, 789 (2005).
[42] C. S. Fadley, Synchrotron Radiation Research: Advances in Surface Science (Plenum, New York, 1990).

[43] C. S. Fadley, Phys. Scr. 1987, 39 (1987).

[44] A. X. Gray, J. Minar, L. Plucinski, M. Huijben, A. Bostwick, E. Rotenberg, S.-H. Yang, J. Braun, A. Winkelmann, G. Conti, D. Eiteneer, A. Rattanachata, A. A. Greer, J. Ciston, C. Ophus, G. Rijnders, D. H. A. Blank, D. Doennig, R. Pentcheva, J. B. Kortright, C. M. Schneider, H. Ebert, and C. S. Fadley, Europhys. Lett. 104, 17004 (2013).

[45] T. Straub, R. Claessen, P. Steiner, S. Hüfner, V. Eyert, K. Friemelt, and E. Bucher, Phys. Rev. B 55, 13473 (1997).

[46] E. J. Moon, J. M. Rondinelli, N. Prasai, B. A. Gray, M. Kareev, J. Chakhalian, and J. L. Cohn, Phys. Rev. B 85, 121106 (2012).

[47] M. J. Han, C. A. Marianetti, and A. J. Millis, Phys. Rev. B 82, 134408 (2010).

[48] A. Blanca-Romero and R. Pentcheva, Phys. Rev. B 84, 195450 (2011).

[49] M. Hepting, M. Minola, A. Frano, G. Cristiani, G. Logvenov, E. Schierle, M. Wu, M. Bluschke, E. Weschke, H.-U. Habermeier, E. Benckiser, M. Le Tacon, and B. Keimer, Phys. Rev. Lett. 113, 227206 (2014).

[50] G. Berner, A. Müller, F. Pfaff, J. Walde, C. Richter, J. Mannhart, S. Thiess, A. Gloskovskii, W. Drube, M. Sing, and R. Claessen, Phys. Rev. B 88, 115111 (2013). 